\title{
Determinants of intermittent preventive treatment with sulfadoxine-pyrimethamine in pregnant women (IPTp-SP) in Mali, a household survey
}

Oumar Sangho ${ }^{1,2^{*}} \mathbb{D}$, Moctar Tounkara ${ }^{2}$, Lillian Joyce Whiting-Collins ${ }^{3}$, Madeleine Beebe ${ }^{3}$, Peter J. Winch ${ }^{3}$ and Seydou Doumbia ${ }^{2}$

\begin{abstract}
Background: In malaria endemic regions, intermittent preventive treatment with sulfadoxine-pyrimethamine (IPTp$\mathrm{SP}$ ) is recommended for all pregnant women during prenatal consultation, from the fourth month of pregnancy up to the time of delivery. The Government of Mali is aiming for universal coverage of IPTp-SP. However, coverage is still low, estimated to be $18 \%$ for completion of three doses (IPTp-SP3). The objective of this study was to identify the factors that influence IPTp-SP adherence in the Health District of Sélingué, Mali.

Methods: This was a cross-sectional survey with 30 clusters, proportional to village size, with two stages of sampling. Data were collected electronically with Magpi software during face-to-face interviews/surveys. The data were analysed with SPSS version 20. A descriptive analysis and bivariate and multivariate logistic regression were performed. An equity analysis examined the effect of distance from health care facility on completion of three or more antenatal visits $(\mathrm{ANC} 3+$ ) and three or more doses of intermittent preventive treatment (IPTp-SP3 + ).

Results: Of the 1,021 women surveyed, $87.8 \%(n=896)$ attended at least one ANC visit. Of these, $86.3 \%(n=773)$ received at least one dose of IPTp-SP. Compliance with three or more doses of IPTp-SP was 63.7\%. The determinants statistically related to $A N C 3$ + were the early initiation of $A N C(O R=3.22[1.22,10.78])$, and the presence of a community health centre $(\mathrm{CHC})$ in the village $(\mathrm{OR}=9.69[1.09,86.21])$. The ability to read $(\mathrm{OR}=1.60[1.01,2.55])$, the early initiation of $A N C(O R=1.46[1.06,2.00]$, knowledge of the utility of the drug $(O R=2.38[1.24,4.57])$, and knowledge of the recommended dose of the drug $(\mathrm{OR}=6.11[3.98,9.39])$ were related to completion of three or more treatments (IPTp-SP3+).

Conclusion: The early initiation of ANC was a positive determinant of the completeness of both ANC3 + and IPTp-SP3 + . This study shows that a successful implementation of the IPTp strategy can be achieved by improving access to prenatal care at community health facilities, and strengthening patient-provider communication to ensure adequate knowledge on dosing of IPTP-SP and the benefits to mother and child.
\end{abstract}

\footnotetext{
*Correspondence: osangh2005@gmail.com

${ }^{1}$ Department of Education and Research of Biological and Medical

Sciences, Faculty of Pharmacy, University of Sciences, Techniques and Technologies of Bamako, Bamako, Mali

Full list of author information is available at the end of the article
}

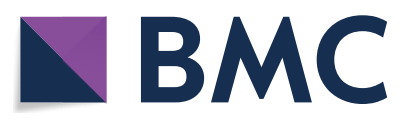

(c) The Author(s) 2021. This article is licensed under a Creative Commons Attribution 4.0 International License, which permits use, sharing, adaptation, distribution and reproduction in any medium or format, as long as you give appropriate credit to the original author(s) and the source, provide a link to the Creative Commons licence, and indicate if changes were made. The images or other third party material in this article are included in the article's Creative Commons licence, unless indicated otherwise in a credit line to the material. If material is not included in the article's Creative Commons licence and your intended use is not permitted by statutory regulation or exceeds the permitted use, you will need to obtain permission directly from the copyright holder. To view a copy of this licence, visit http://creativeco mmons.org/licenses/by/4.0/. The Creative Commons Public Domain Dedication waiver (http://creativecommons.org/publicdomain/ zero/1.0/) applies to the data made available in this article, unless otherwise stated in a credit line to the data. 
Keywords: Access to care, Geographic access, Intermittent preventive treatment, Malaria in pregnancy, Antenatal care

\section{Background}

Malaria is a major public health problem. In 2019, the number of malaria cases was estimated at 229 million [1]. Of these cases, $94 \%$ were recorded by the World Health Organization (WHO) Africa region, followed by the South-East Asia region (3\%) [1]. Of the 409,000 malaria deaths worldwide, $51 \%$ were recorded in seven African countries [1]. Malaria is especially detrimental to the health and well-being of women and children [2]. It can lead to maternal anaemia, which increases the risk of haemorrhage during childbirth [2]. In some endemic areas, malaria can directly contribute up to $25 \%$ of all maternal death [2]. Malaria during pregnancy also increases the risk of miscarriage and affects foetal development, as well as preterm birth and low birth weight [3]. In 2019, 33 million pregnancies were estimated in malaria-endemic areas in Africa, of which 35\% (12 million) were exposed to malaria infection [1].

As part of antenatal care (ANC) services, every effort should be made to improve access to sulfadoxinepyrimethamine intermittent preventive treatment (IPTp$\mathrm{SP})$ everywhere in Africa where malaria endemicity level ranges from moderate to high [4-6]. The WHO recommends at least four ANC visits during pregnancy $[4,6]$. Starting as early as possible in the second trimester, IPTp$\mathrm{SP}$ is recommended for all pregnant women at each ANC visit until delivery, and doses should be administered at least one month apart [6]. Sulfadoxine-pyrimethamine (SP) should not be administered during the first trimester of pregnancy [6]. The last dose of IPTp-SP can be administered up to the time of delivery without any safety concern $[4,6]$. IPTp-SP should preferably be administered in the form of three SP tablets, under direct observation, which is the total required dosage of $1500 \mathrm{mg} / 75 \mathrm{mg}$, and can be administered with or without food [6]. SP should not be given to women receiving prophylactic treatment with cotrimoxazole because of a higher risk of adverse events [6].

Globally, the percentage of women receiving IPTp-SP during pregnancy has increased over the years, although it remains below targets of $80 \%$ as cited in Yaya et al. [7] and below the target of universal coverage of all pregnant women attended ANC services [8]. IPTp-SP has been adopted by 37 countries [8]. In 2019, $80 \%$ of pregnant women used ANC services at least once. The percentage of pregnant women who received IPTp-SP1+, IPTp-SP2 + and IPTp-SP3 + were 62\%, $49 \%$ and $34 \%$ respectively [1]. A study using data from the Malaria
Indicator Surveys (MIS) conducted in eight Sub-Saharan African countries found the overall prevalence of $29.5 \%$ (95\% CI $=28.2-30.5)$ for IPTp-SP3 + in 2018 [7].

In Mali, the objective of the National Policy for Malaria Control, led by the national malaria control programme ('Programme National de Lutte contre le Paludisme', PNLP), is to achieve universal coverage of SP through free distribution to pregnant women countrywide [9]. The main goal of the 2013-2017 Strategic Plan of the PNLP [5] was to ensure universal access to prevention measures for $100 \%$ of the population at risk of malaria, including the use of IPT by pregnant women [5].

In the Malian healthcare system, ANC provides women with the opportunity to get IPTp-SP at no cost. In 2014, $74 \%$ of Malian pregnant women attended ANC1 while $41 \%$ attended ANC4 and above (ANC4+) [10]. In the Sikasso region, there was $79 \%$ coverage for ANC1 [10]. In Sélingué, in 2017, the frequencies of ANC1, ANC3 and ANC4 were $89 \%, 26 \%$ and $28 \%$, respectively [11].

Hurley's secondary analysis of the 2012-2013 Demographic and Health Survey (DHSM-V) data [10] shows that $56.2 \%$ and $29.9 \%$ of recently pregnant women benefited from IPTp-SP1 and TPIg- SP2 +, respectively, in Mali [12]. The MIS conducted in Mali in 2015 shows adherence of $66.4 \%$ for IPTp-SP1, 44.4\% for IPTp-SP2 and $21 \%$ for IPTp-SP3 + [13].

In Mali's Sikasso Region, the DHS found adherence of IPTp-SP1 to be $68.42 \%$ [14]. Adherence IPTp-SP2 and IPTp-SP3 + were $50.8 \%$ and $26.8 \%$, respectively [14]. In 2015 , the MIS showed an adherence of $68.7 \%, 44.4 \%$ and $22.2 \%$, respectively for IPTp-SP1, IPTp-SP2 and IPTpSP3 + [13]. In Sélingué, in 2016, $72 \%$ of pregnant women had received IPTp-SP1 compared to $43 \%$ for IPTp-SP2 and only $19 \%$ for IPTp-SP3 [11].

Based on these data, the goals of the PNLP have not been achieved.

Several studies have explored the determinants of the use and completeness of IPTp-SP [12, 15-31]. Nonattendance and/or completeness of ANC is considered a key determinant of IPTp-SP coverage [11, 15, 20, 24]. A qualitative study conducted in Mali in 2013 identified the following factors influencing the completeness of two doses of IPTp-SP: late use or non-use of ANC services, perception of malaria during pregnancy, poor acceptability of SP, stock-outs of SP, and insufficient information on the policy of providing SP free of cost to pregnant women [15]. In spite of this free IPTp national distribution policy, the authors reported variations in IPTp costs at various 
health facilities and during prenatal visits [15]. Lack of education and economic wellbeing have also been identified as factors influencing the use of IPTp-SP, with high level of education, high level of economic well-being, and living in an urban area strongly associated with women taking IPTp-SP [23]. In 2013, a meta-analysis was done by Hill et al. [24]. The results suggested that barriers to the use of IPTp-SP included lack of clarification of guidelines and policy, poor organization of services, stockouts, costs of health services, lack of competent health workers, and the underuse of ANC by women [24]. Key determinants of IPTp-SP were level of education, knowledge of malaria and IPTp-SP, socio-economic status, parity, and number of and early initiation of ANC visits [24]. Despite these factors affecting completeness of IPTp-SP, frequencies remain low. It is necessary to find an alternative strategy to improve IPTp-SP coverage. Sélingué is a malaria endemic, irrigated rice-growing area with a malaria incidence of $16.9 \%$ [11]. This study was conducted to identify the factors that specifically influence IPTp-SP in the Sélingue Health District in an effort to inform local efforts to improve IPTp-SP.

\section{Methods}

\section{Site}

The study was conducted in 2016, from January $11^{\text {th }}$ to February $9^{\text {th }}$ in the health district of Sélingué, located $120 \mathrm{~km}$ southwest of Bamako. The health district of Sélingué is composed of seven subdistricts: Kangaré, Binko, Siékorolé, Tanga, Carrière, Diarani and Faraba and has three private clinics. The population in 2012 was 91,425 across 60 villages [32]. The dam on the Sankarani River, a branch of the Niger River, has created an artificial lake of $409 \mathrm{~km}^{2}$ in Sélingué, making rice cultivation, gardening, and fishing the main activities in the area [33]. In 2016, there were 18 community health workers $(\mathrm{CHW})$ in the district, each working with several community relays (CR) and traditional birth attendants in each village [34]. CHWs were involved in minor medical care for children under five years of age and in the education and referral of pregnant women to ANC services.

\section{Design}

This was a cross-sectional survey with cluster sampling proportional to village population size at two levels. Thus, the number of clusters per village depended on the population size in each village. A total of 30 clusters were needed to have a representative sample. Overall, 960 households were selected for the 30 clusters (each cluster consisted of 32 households with at least one eligible woman). The minimum sample size was 981 households. To be eligible, the woman must have had a pregnancy in the two years prior to the survey, regardless of pregnancy outcome, according to the same selection process used in the MIS and the sixth Demographic and Health Surveys (DHS-VI) $[13,14]$. Participants were interviewed face-toface by interviewers and were shown the three SP tablets to see if they recognized them.

\section{Data quality management and control}

All data were collected on tablets by six data collectors and two supervisors (enumerators) over one month, using Magpi remote data collection software [35]. Data collectors were trained by the supervisors. The tools were pre-tested in Diago, a village near Bamako. Data were checked by the enumerators, uploaded to a secure cloudbased server, and later exported to an Excel spreadsheet by the data manager. Data were then analysed in SPSS software version 20 [36].

\section{Data analysis}

Analysis focused on the following two outcomes:

\section{Completion of three or more antenatal consultations (ANC3+)}

Women who completed at least three ANC visits were coded 1 , and those who did not were coded 0 . These women should have received IPTp-SP1+(Fig. 1). Only women who achieved ANC3 + were considered, because with the current national policy, a woman may have obtained all three doses of IPTp-SP without completing the ANC4+.

\section{IPTp-SP3 + intake}

Women who received at least three doses of IPTp-SP were coded 1 , and 0 if they did not receive the three doses. These women were those who have completed their ANC3 + (Fig. 1).




Bivariate analyses were conducted according to the flow presented in Fig. 1. Bivariate analysis for the outcome of IPTp-SP3 + intake included women who completed ANC1, IPTp-SP1 and ANC3 + and was performed using Chi square tests and simple logistic regression. Odds ratios (OR) were estimated with 95\% confidence intervals $(95 \% \mathrm{CI})$ and the association between dependent and independent variables (age, level of education, parity, distance from the village to $\mathrm{CHC}$, literacy, age of pregnancy at the first ANC, knowledge of the usefulness of SP, knowledge of the recommended SP dose, knowledge of the period of starting taking SP, number of SP payments, given ITN in ANC, presence of $\mathrm{CHC}$ in the village, rurality of the village of residence, poverty quintile) was verified using the Kendall tau B correlation [37]. Variables that had a significant association during the bivariate analysis were selected for the multivariate (global) model. Multiple logistic regression was performed and adjusted odds ratios (ORa) were presented with a p value of 0.05 . Correlation between the independent variables with Kendall's tau B was assessed and variables that demonstrated significant correlations were eliminated. For ANC3+, the variable "ability to read", due to its importance for understanding of medical information, was added.

To measure equity in terms of geographic accessibility to facilities providing ANC, the Gini index and the concentration curve (CC) were used. These data analysis tools are recognized as standard measures employed by health economists to estimate wealth inequity on various health indicators [38-45]. These tools were adapted in this study to estimate inequities in health services utilization according to geographic accessibility. Geographic accessibility was measured using the distance index between a woman's village of residence and the CHC. This index places women's villages of residence individually on a continuous scale of relative distance. Villages have been grouped into five distance quintiles. Distance quintiles were used to compare the influence of distance on ANC3+ and IPTp-SP3+. Principal component and factor analysis were used to determine quintiles of poverty. The original line (oblique or diagonal) shows perfect equity. The more the second line curves away from the perfect equity line, the higher the degree of inequity. A curve below the equity line indicates a disproportionate use of services for households from villages close to health facilities. Although the concentration curve is a useful tool for the graphical representation of inequity, it does not quantify the magnitude of inequity. Hence the use of The Gini Index, which is a quantitative measure of inequity in the use of health care. It was used by Wagstaff \& van Doorslaer in 2004 to measure the degree of inequity associated with household wealth [45]. The value of the index varies between -1 and +1 . A value of
0 indicates that the use of health services is equitably distributed among socio-economic groups [46]. In this case, the confidence interval around the index value includes zero. If zero is not within the confidence interval, there is a statistically significant inequity in the use of health services [46]. A negative value of the concentration index implies greater use among the more remote health facilities while a positive value indicates that women in villages around health facilities have greater coverage than women far from health facilities.

\section{Results}

The analysis included 1021 women. Table 1 shows the socio-demographic characteristics of the study sample. More than a quarter of women surveyed (26.2\%) were between 20-24 years old. Many women (68.7\%) had no level of education. Multiparas were the most frequent with $61.1 \%$. Most of the women surveyed (73.4\%) lived within $5 \mathrm{~km}$ of a CHC. Figure 1 shows the flow chart of the completeness of ANC3+and IPTp-SP3+. Among the 447 women who completed IPTp-SP3+, about half (223) took SP under Direct Observed Therapy (DOT). One hundred of those women said they brought their own water to the centre for the DOT. Only 96 of the 223 affirmed they took SP on empty stomach. Among the

Table 1 Sociodemographic characteristics of women surveyed in 2016 Sélingué District, Mali ( $N=1021)$

\begin{tabular}{lcc}
\hline Sociodemographic Variables & $\mathbf{n}$ & $\%$ \\
\hline Age groups & 247 & 24.2 \\
$15-19$ & 268 & 26.2 \\
$20-24$ & 212 & 20.8 \\
$25-29$ & 154 & 15.1 \\
$30-34$ & 103 & 10.1 \\
$35-39$ & 37 & 3.6 \\
$40-49$ & & \\
Level of education & 701 & 68.7 \\
No level of education & 213 & 20.9 \\
Primary 1 & 88 & 8.6 \\
Primary 2 & 19 & 1.9 \\
At least secondary level & & \\
Parity & 199 & 19.5 \\
Primipara & 197 & 19.3 \\
Secondi parous & 625 & 61.2 \\
Multiparous & & \\
Geographic accessibility & & 73.4 \\
Distance from the village to CHC & 749 & 16.6 \\
0 to 5 km & 169 & \\
6 to 15 km & 103 & \\
$>15$ km & & \\
\hline
\end{tabular}


other half (224) who carried their SP home, 215 (96\%) said they took it immediately when they got home.

Sixty five percent of women who completed IPTpSP3 + mentioned that they were asked if they had eaten before coming to the centre, and $78.1 \%$ affirmed the availability of water for the DOT.

\section{Bivariate and multivariate analysis}

Tables 2 and 3 present results from the bivariate and multivariate analyses for ANC3+ and IPTp-SP3+. Kendall tau $\mathrm{B}$ analysis revealed a negative correlation $(-0.048)$ between the presence of CHC in the woman's village of residence and the rurality of the village of residence, $\mathrm{p}=0.175$. There was a positive correlation $(0.131)$ between knowledge of the recommended SP dose and knowledge of when to start taking SP, $\mathrm{p}<0.0001$. There was a positive correlation (0.432) between knowledge of the recommended SP dose and the number of payments for SP, $\mathrm{p}<0.0001$. Knowledge of the recommended dose was correlated to two variables. Therefore, it was removed from the multivariate analysis presented in Table 2. Similar analysis for IPTp-SP3 + was performed. There was a positive correlation (0.121) between knowledge of the benefit of taking SP and the knowledge of the recommended dose, $\mathrm{p}=0.001$. There was a positive correlation (0.441) between knowledge of the recommended doses of SP and the number of payments, $\mathrm{p}<0.0001$. Therefore, knowledge of the recommended dose was removed from the multivariate analysis for IPTp-SP3 + from Table 3.

\section{Equity and distance to $\mathrm{CHC}$}

Figures 2 and 3 present the results of the equity analysis for completeness of adherence to ANC3+and IPTp-SP3 + under the influence of distance from women's home villages to the $\mathrm{CHC}$. Women close to the $\mathrm{CHC}$ were more likely to complete $\mathrm{ANC} 3+$ and IPTp$\mathrm{SP} 3$ + than those far from the $\mathrm{CHC}$, although this inequity was not statistically significant (Table 4 ).

Table 2 Bivariate and multivariate analysis: Predictive variables of ANC3 + among women surveyed in 2016, Sélingué, Mali

\begin{tabular}{|c|c|c|c|c|}
\hline Characteristics & n & $\mathrm{ANC3}+\mathrm{n}(\%)$ & OR $[\mathrm{Cl} 95 \%]$ & ORa [Cl 95\%] \\
\hline Can read & 773 & & & \\
\hline No & 661 & 599 (90.6) & - & 1 \\
\hline Yes & 112 & $103(92.0)$ & - & $1.04[0.2 ; 5.56]$ \\
\hline Age of pregnancy at the first ANC & 757 & & & \\
\hline 4 months or more & 444 & $385(86.7)$ & 1 & 1 \\
\hline 3 months or less & 313 & $306(97.8)$ & $6.7^{* * *}[3.02 ; 14.88]$ & $3.62 *[1.22 ; 10.78]$ \\
\hline Knowledge of the recommended SP dose & 773 & & & \\
\hline Incorrect answer & 538 & $476(88.5)$ & 1 & - \\
\hline Know the recommended dose & 235 & $226(96.2)$ & $3.27^{* * *}[1.6 ; 6.7]$ & - \\
\hline Knowledge of the period of starting taking SP & 773 & & & \\
\hline Incorrect answer & 665 & $597(89.8)$ & 1 & 1 \\
\hline 4 months or less & 108 & $105(97.2)$ & $3.99 *[1.23 ; 12.91]$ & $5.41[0.67 ; 43.93]$ \\
\hline SP payment number & 280 & & & \\
\hline 1 time & 112 & $84(75.0)$ & 1 & 1 \\
\hline 2 times & 71 & 70 (98.6) & $23.33^{* *}[3.1 ; 175.84]$ & $21,5^{* *}[2.64 ; 175.09]$ \\
\hline 3 times or more & 97 & $95(97.9)$ & $15.83^{* * *}[3.66 ; 68.47]$ & $11,24^{* *}[2.5 ; 50.46]$ \\
\hline Given ITN in ANC & 772 & & & \\
\hline No & 210 & $183(87.1)$ & 1 & 1 \\
\hline Yes & 562 & $519(92.3)$ & $1.78^{*}[1.07 ; 2.97]$ & $1,51[0.62 ; 3.7]$ \\
\hline Presence of $\mathrm{CHC}$ in the village & 773 & & & \\
\hline CHC planned but not operational & 22 & $17(77.3)$ & 1 & 1 \\
\hline Village with $\mathrm{CHC}$ & 282 & $257(91.1)$ & $3.02^{*}[1.03 ; 8.89]$ & $9,69 *[1.09 ; 86.21]$ \\
\hline No planned or operational CHC & 469 & $428(91.3)$ & $3.07^{*}[1.08 ; 8.75]$ & $7,8[0.98 ; 62.4]$ \\
\hline Rurality of the village of residence & 773 & & & \\
\hline Rural & 448 & $420(93.8)$ & 1 & 1 \\
\hline Less rural & 325 & $282(86.8)$ & $0.44^{* *}[0.27 ; 0.72]$ & $0.69[0.27 ; 1.77]$ \\
\hline
\end{tabular}

${ }^{*}=\mathrm{p}<0,05 ;{ }^{* *}=\mathrm{p}<0,01 ;{ }^{* * *}=\mathrm{p} \leq 0,001$

$\mathrm{OR}=$ Odds Ratio. ORa $=$ Adjusted Odds Ratio 
Table 3 Bivariate and multivariate analysis: Predictive variables of IPTp-SP3 + among women surveyed in 2016, Sélingué, Mali

\begin{tabular}{|c|c|c|c|c|}
\hline Characteristics & $\mathrm{n}$ & IPTp-SP3 + n (\%) & OR [Cl 95\%] & ORa [Cl 95\%] \\
\hline Poverty quintile & 697 & & & \\
\hline Q1 (poorest) & 128 & $85(66.4)$ & 1 & 1 \\
\hline Q2 (poorer) & 140 & $92(65.7)$ & $0.97[0.58 ; 1.61]$ & $1.5[0.24 ; 9.25]$ \\
\hline Q3 (mean) & 142 & $100(70.4)$ & $1.20[0.72 ; 2.01]$ & $1.23[0.24 ; 6.15]$ \\
\hline Q4 (wealthier) & 146 & $79(54.1)$ & $0.60^{*}[0.37 ; 0.97]$ & $0.17^{*}[0.03 ; 0.92]$ \\
\hline Q5 (most wealthy) & 141 & $87(61.7)$ & $0.82[0.49 ; 1.34]$ & $0.48[0.09 ; 2.54]$ \\
\hline Can read & 701 & & & \\
\hline No & 599 & $373(62.3)$ & 1 & 1 \\
\hline Yes & 102 & $74(72.5)$ & $1.60^{*}[1.01 ; 2.55]$ & $10.27^{*}[1.52 ; 69.28]$ \\
\hline Age of pregnancy at the first ANC & 690 & & & \\
\hline 4 months or more & 384 & $229(59.6)$ & 1 & 1 \\
\hline 3 months or less & 306 & $209(68.3)$ & $1.46^{*}[1.06 ; 2.00]$ & $1.58[0.53 ; 4.72]$ \\
\hline Knowledge of the usefulness of SP & 700 & & & \\
\hline Incorrect answer & 641 & $399(62.2)$ & 1 & 1 \\
\hline Prevention of malaria & 59 & $47(79.7)$ & $2.38^{* *}[1.24 ; 4.57]$ & $1.32[0.2 ; 8.76]$ \\
\hline Knowledge of the recommended SP dose & 701 & & & \\
\hline Incorrect answer & 475 & $250(52.6)$ & 1 & - \\
\hline Know the recommended dose & 226 & $197(87.2)$ & $6.11^{* * *}[3.98 ; 9.39]$ & - \\
\hline SP payment number & 249 & & & \\
\hline 1 time & 84 & $16(19.0)$ & 1 & 1 \\
\hline 2 times & 70 & $66(94.3)$ & $70.13^{* * *}[22.28 ; 220.76]$ & $139.18^{* * *}[32.94 ; 588.06]$ \\
\hline 3 times or more & 95 & $92(96.8)$ & $130.33^{* * *}[36.52 ; 465.18]$ & $244.73^{* * *}[54.28 ; 1103.5]$ \\
\hline Rurality of the village of residence & 701 & & & \\
\hline Rural & 419 & $287(68.5)$ & 1 & 1 \\
\hline Less rural & 282 & $160(56.7)$ & $0.60^{* *}[0.44 ; 0.82]$ & $0.17^{* *}[0.05 ; 0.57]$ \\
\hline
\end{tabular}

${ }^{*}=\mathrm{p}<0,05 ;{ }^{* *}=\mathrm{p}<0,01 ;{ }^{* * *}=\mathrm{p} \leq 0,001$

OR Odds Ratio, Ora Adjusted Odds Ratio

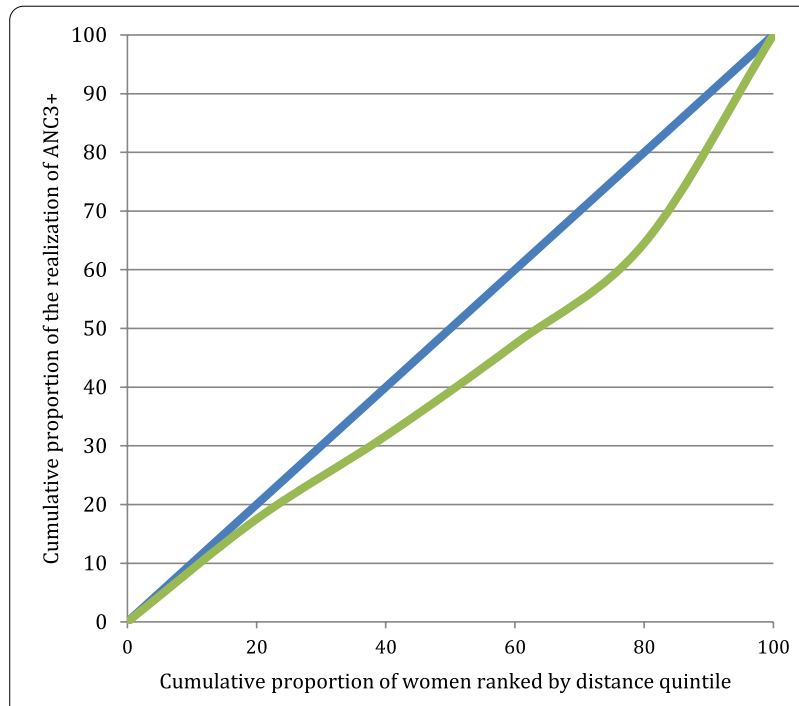

Fig. 2 Curve of concentration of the realization of $\mathrm{ANC}^{+}+$in the women surveyed in 2016, Sélingué, Mali

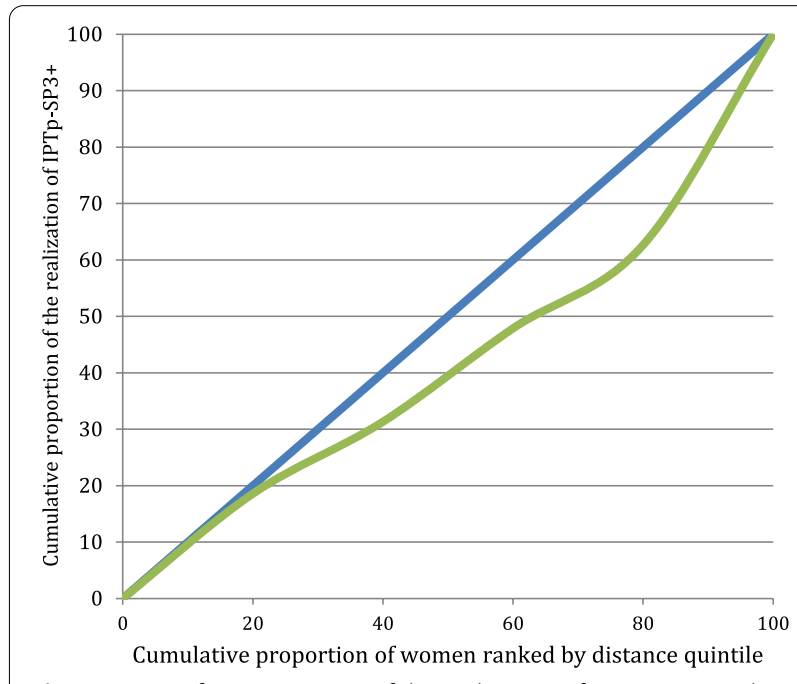

Fig. 3 Curve of concentration of the realization of IPTp-SP3 + in the women surveyed in 2016, Sélingué, Mali 
Table 4 Concentration index of the realization of ANC3 + and IPTP-SP3 + in the women surveyed in 2016, Sélingué, Mali

\begin{tabular}{|c|c|c|c|c|}
\hline $\begin{array}{l}\text { Concentration index } \\
\text { (CIndex) }\end{array}$ & Variance of CIndex & Standard error & t Statistic & 95\% Confidence interval (Cl) \\
\hline \multicolumn{5}{|l|}{$\mathrm{ANC3}+$} \\
\hline 0.1641 & 0.0072 & 0.0850 & 1.9306 & {$[-0.0025 ; 0.3307]$} \\
\hline \multicolumn{5}{|l|}{ IPTp-SP3+ } \\
\hline 0.1584 & 0.0096 & 0.0980 & 1.6168 & {$[-0.0336 ; 0.3504]$} \\
\hline
\end{tabular}

\section{Discussion}

The same selection process of women as in the MIS and the DHS-V was used $[10,13]$. ANC3 + as a dependent variable was chosen because from three ANC visits it is possible to have an adherence of IPTp-SP3 + [4-6]. This choice allowed women who had missed opportunities to benefit from IPTp-SP to be excluded from the determinants analysis, as Hurley reported [12].

\section{Frequency of ANC3 + and IPTp-SP3 +}

ANC is the entry point for the women included in this study to benefit from the WHO recommended intervention package $[4,6,38]$ for their protection, and that of their foetus, as concluded the study by Hill et al. [16] and others [12, 47]. The frequency of ANC1 $+(87.8 \%)$ in this study was high compared to the $78.5 \% \mathrm{ANC} 1+$ found in the fifth DHS of Mali, in the Sikasso region [14], and the $78.3 \%$ found in the meta-analysis of van Eijk et al. [23]. It is also higher than the $81 \%$ in Hill et al. [16]. However, it is similar to the $89 \%$ of the 2016 statistical yearbook [11]. The frequency of ANC3 + (90.8\%) in this study was high compared to those found in some studies $[11,14,16,23]$.

The study found an IPTp-SP3 + frequency of $63.7 \%$ among women who completed ANC3 +, which is similar to the frequency that Muhumuza et al. found in Uganda [29]. This frequency was greater than the $38.5 \%$ found by Odjidja et al. [48], but less than the $71 \%$ found by Ibrahim et al. [49], both in Ghana in 2017. Furthermore, it is more than the frequencies of the 2015 MIS of 19\% in Sikasso [13], the $19.1 \%$ of the 2016 statistical yearbook [11], and the frequencies found in other studies $[12,16,23,25,26$, $29,46,50,51]$. For instance, Hill et al. found $28.6 \%$ for IPTp-SP2 + and 47.8\% for IPTp-SP1 in Segou, Mali [16].

The high frequency of IPTp-SP is related to several factors. Sélingue District is an area that has benefited from the presence of a research unit of the National Institute of Research in Public Health and the intervention of NonGovernmental Organizations (NGOs), including Borne Fonden. The implementation of the project "Integrated control of malaria based on an environmental and community base in agro-ecosystems of West Africa" in 2012 and 2013 is also an influencing factor [52]. During this project, an awareness raising campaign on ANC was conducted through local radio stations [52]. Additionally, the Village Malaria Committees and Farmers Field school served as a means for information, education, and action [52]. The presence of CHWs and Village Health Volunteers (VHV) have also helped to raise awareness. Notably, in 2014, the CHWs conducted 1,660 home visits involving 5,464 people (69.6\% of whom were women), 1,369 discussion and sharing sessions, and 544 counseling sessions for 16,486 people ( $82 \%$ of whom were women) [53].

Since the implementation of the policy of three or more doses of SP started in Sélingué in 2014, it is possible that the women surveyed obtained the number of doses they mentioned [6]. Many women surveyed recognized the three SP tablets that the investigators showed them. Some of them used its local name (sumaya fura kisè saaba). This recognition was not always followed by the awareness of its usefulness. The investigators did not say the name in advance, to make sure the woman recognized the pills. Women's assertions based on the Kappa concordance test obtained by Hill et al. in Segou in 2014 were considered [16]. After the current study, the WHO guidance changed from "at least four ANC visit" to "eight visits" $[4,6]$. This change could influence future studies on the number of IPTp-SP.

This study may have also benefited from the policy shift in IPTp-SP, with the possibility of taking the medication up to the time of delivery, starting at four months of pregnancy. This could be an explanation of the observed frequency. Moreover, this is the first study that covered the whole district of Sélingué.

\section{Determinants of ANC3+}

The participants who reported knowledge of the usefulness of taking SP and had started taking SP earlier in their pregnancies were more likely to benefit from ANC3 + compared to those who did not know of SP's usefulness or who did not take it early in pregnancy. This theory is reinforced by the education and literacy level. Others, including Webster et al., and Faye et al., reported education as a predictor of ANC compliance in their studies $[17,21]$.

Distance to facility was a barrier to the completeness of ANC3 $+[12,54,55]$. This is why women in villages with 
a $\mathrm{CHC}$ were significantly more likely to achieve their $\mathrm{ANC} 3+$ as compared to those in villages without a $\mathrm{CHC}$. An analysis of data from 10 West African countries in 2016 found that distance, in addition to poverty, level of education, and rurality of the village of residence, were barriers to ANC utilization, as well as its effectiveness [56]. Additionally, the 2016 Statistical Yearbook identified the following factors to explain low rates of ANC: the late initiation of reproductive health services, inadequate completion of monitoring forms, insufficient qualified staff in peripheral reproductive health services, insufficient active research, inadequate reception, and poor communication [11].

\section{Determinants of the observance of IPTp-SP3 +}

The $12.2 \%$ of women who did not have any antenatal visits would not receive IPTp-SP. Among those who had received ANC, 13.5\% had not received SP. ANC appears as a fundamental barrier to the completeness of IPTp$\mathrm{SP} 3+$. Furthermore, not receiving ANC was identified as a barrier to the completeness of IPTp-SP3 + in a study conducted by Hurley et al. in 2016 [12]. Similarly, a study by Sangaré et al. found that $68.7 \%$ of women eligible for IPTp-SP2 did not take any or simply had a single dose of IPTp-SP [19]. Yaya et al. found that women who completed ANC4 + had higher odds of taking IPTp-SP drugs $(\mathrm{OR}=1.656,95 \% \mathrm{CI}=1.194-2.299)$ [57]. Similar result was found by Mushi et al. [58].

The results show that relatively wealthy women were significantly (40\%) less likely to complete IPTp$\mathrm{SP} 3+$ compared to poor women, as opposed to what is generally reported in the literature [27], where adherence is proportional to wealth level. One explanation of this may be that more wealthy women attend private clinics for their ANC.

Women who were educated, who knew the usefulness of SP and the correct dose of SP were significantly more likely to complete IPTp-SP3 +. Similar findings were reported by Hill et al. in Mali, Ameh et al. and Onyeneho et al. in Nigeria, Kibusi et al. and Mushi et al. in Tanzania, and Yaya et al. in Ivory Coast [16, 25, 27, 57-59]. The compliance with IPTp-SP3 +, therefore, appears proportional to the level of education. In their systematic review and meta-analysis, Hill et al. found that having the correct information on IPTp-SP increased the likelihood of taking the SP [24]. In the same way, Pell et al. found that lack of knowledge was a barrier to IPTp-SP [60].

This study found that starting ANC early was a good indicator of IPTp-SP3 + compliance, which reinforces findings from other studies [16, 24, 27]. However, with the WHO recommendations [6], a woman can have three doses (IPTp-SP3+) even if she started her ANC at 6-7 months, taking SP every month, provided there is regular attendance at ANC visits after this late start. In this case, this indicator could, in the future, no longer serve as an adequate indicator of compliance.

The DOT of SP, as recommended by WHO and PNLP [4-6], was not widely practiced in Sélingué, compared to findings of other studies $[26,61]$. Half of the participants this study had practiced the DOT of SP. The other half took their SP home for self-administration, in absence of an observer, contrary to the recommendations [4-6]. The absence of observed treatment represents a barrier to IPTp-SP3 + compliance [16, 17, 24-26].

Few women were aware that SP can be taken on an empty stomach. Unavailability of drinking fountain or cups by the fountain, as well as asking women if they had eaten prior to coming to the health centre, influenced the non-observance of DOT of IPTp-SP. This suggests lack of knowledge of health workers on the guidelines for the administration of SP as identified by van Eijk et al. [23]. Similarly, Mubyazi et al. [62] found that the lack of clean water and cups were barriers to the DOT of SP. In another study, some pregnant women brought their own water bottle to the health centre to take SP, which enabled a barrier to DOT of SP [26]. Other authors found the positive influence of the availability of drinking water on the DOT of SP $[17,28]$, hence the availability of clean water and cups is recommended to improve the DOT [50]. Additionally, as noncompliance with DOT of IPTpSP guidelines has been identified in several studies [12, $15,16,19,24]$, a need for competence building is largely suggested [18, 24, 27, 63].

Distance was also identified as a barrier to IPTp-SP3+, and was confirmed by the equity analysis. Women in $\mathrm{CHC}$ village sites were more likely to complete IPTp$\mathrm{SP} 3+$ compared to women in villages far from the $\mathrm{CHC}$. These findings are supported by those in the study by Hurley et al. [12]. However, the findings from this study conflict with those of Hill et al. which suggests that women in villages close to $\mathrm{CHC}$ were less likely to complete IPTp-SP [16].

Purchasing SP was a facilitator of IPTp-SP3+completeness. This trend was confirmed by the number of payments mentioned by the participants. The higher the number of payments for SP, the more likely the woman was to complete IPTp-SP3 + , which appeared paradoxical in the local context. The national guidelines suggest that SP must be delivered to pregnant women free of charge $[4,6,64]$. However, paying for SP did not appear as a barrier. Payment of SP was reported by women in the study carried out by Klein et al. [15] in Koulikoro and Sikasso, Mali, which support the comments made by the participants in this study. Klein et al. found that payment was one barrier for the IPTp$\mathrm{SP} 2+$ and that even staff were confused about IPTp-SP 
being free of charge [15]. Payment for SP linked to IPTp-SP completeness may be due to knowledge of its usefulness, which was observed to correlate positively with knowledge of the recommended doses, as the following: 1) the husbands or attendants were in charge of the payment of the prescription given to the women; 2) difficulties to differentiate between paid and free drugs; 3 ) the husbands or attendants did not share with women the information on the policy of offering SP free of cost; 4) lack of clear explanations of free medicines by staff, or 5) free drugs are not available from the ANC agent but rather from the pharmacy. Some of these explanations are corroborated by the studies of Hurley et al. and Hill et al. [12, 24]. It should be noted that the guidelines recommend both predisposing the free SP in the ANC units instead of the pharmacy and, on the other hand, making the separate SP prescription [64]. This is not always done at the CHC.

The wide confidence interval of SP payment number (Table 3) suggests that more exploration for this relationship is needed.

\section{Equity analysis}

Findings for the equity analysis suggested that distance impacts the completeness of ANC3 + and IPTp-SP3 +. The curves (Figs. 2 and 3) indicated inequities in favour of women in the villages closest to the CHCs, especially after the first quintile of distance. Women nearer were more likely to complete ANC3 + and IPTp-SP3 + than those who were distant.

\section{Study limitations}

The IPTp-SP questions did not specify exactly which dose was targeted because it was not standardized on a specific dose, such as the first dose taken or the last dose taken. The woman could answer on any dose. The IPTp-SP questions were asked only to women who said they had received ANC. Those who did not attend ANC could have interesting information, especially multiparas, hence a potential source of selection bias. The cross-sectional nature of the study and the subjective responses of the women surveyed could introduce information biases, not only by the women themselves, but also by the interviewers due to the length of the questionnaire, the time needed for the interview, and some skips or connection problems in the Magpi software used for electronic data collection. Lastly, the availability of SP stock in the health centres was not evaluated. However, few women mentioned SP stock shortage at the $\mathrm{CHC}$ as the reason for not obtaining and taking SP.

\section{Conclusion}

Despite the high frequency of IPTp-SP3 + in this study, Mali is still far from the goal of universal coverage of pregnant women. Several critical factors in achieving this goal were identified. The positive factors include the level of education, the ability to read, the early start of ANC, the knowledge of the usefulness of the drug, the recommended dose of the drug and the observance of DOT at the CHC. Some of the barriers include the distance to the health facility, the lack of implementation of ANC, and the unavailability of water for DOT. This study shows that a successful implementation of the IPTp strategy can be achieved by improving access to prenatal care at community health facilities, and strengthening patient-provider communication, to ensure adequate knowledge on dosing and IPTp-SP benefits to mother and child.

\section{Abbreviations}

ANC: Antenatal care or antenatal visits; $\mathrm{CHC}$ : Community Health Centre; CHW: Community Health Workers; Cl: Confident Interval; CIndex: Concentration index; CR: Community relays / volunteers; DHS: Demographic and Health Surveys (DHS); DOT: Direct Observed Therapy; IPTp-SP: Intermittent Preventive Treatment with Sulfadoxine-pyrimethamine; ITN: Insecticide-Treated Nets; MIS: Malaria Indicators Survey; NGOs: Non-Governmental Organizations; OR: Odds Ratio; ORa: Adjusted Odds Ratio; PNLP: National Malaria Control Programme; SPSS: Statistical Package for the Social Sciences; VHV: Village Health Volunteers; WHO: World Health Organization.

\section{Acknowledgements}

The study authors gratefully acknowledge Dr Dadé Haidara, Chief of Sélingué Referral Health District during the survey and all the health care providers in the Community Health Centres, and the Community Health Workers and surveyors; Dr Craig Tower, Ms Wendy Yang, Ms. Rachel Turkel, Dr Emily Hurley, Dr Ousmane Ly, Professor Hamadoun Sangho, Professor Akory Ag Iknane, Professor Cheick Oumar Bagayoko for their contribution to the review of the dissertation; Dr Lancina Doumbia and Ms Hannah Marker for the translation. We would also like to thank the Fogarty International Center for supporting Oumar Sangho and Moctar Tounkara under Grant D43TW008652.

\section{Authors' contributions}

OS analysed and interpreted data and wrote the manuscript. MT, LWC and $\mathrm{MB}$ and PJW analysed and interpreted data and reviewed the manuscript. SD review the manuscript. All authors read and approved the final manuscript.

\section{Funding}

This study was funded through a NIH R21 Grant from the Fogarty International Center (Grant Number 1R21TW009885-01). The funder had no role in the design of the study and collection, analysis, interpretation of data, or in writing the manuscript.

\section{Availability of data and materials}

The datasets used and/or analysed during the current study are available from the corresponding author on reasonable request.

\section{Declarations}

\section{Ethics approval and consent to participate}

The study protocol was approved by both ethics committees of the Faculty of Medicine, Dentistry and Pharmacy of Mali (N²015/112/CE/FMPOS) and John Hopkins University of Baltimore in the United States (FWA00000287). The research team members were trained in data collection techniques and questionnaires were pre-tested in a village out of the intervention area. Investigators did not collect participants' names or other personal identifiers. Informed 
consent was read in the local language and approval was obtained with a signature of participants. Married women aged 15 and over were considered adults with the legal power to give informed consent, as stated in Malian law.

\section{Consent for publication}

Not applicable.

\section{Competing interests}

The authors declare that they have no competing interests.

\section{Author details}

'Department of Education and Research of Biological and Medical Sciences, Faculty of Pharmacy, University of Sciences, Techniques and Technologies of Bamako, Bamako, Mali. ${ }^{2}$ Department of Education and Research in Public Health and Specialties, Faculty of Medicine and Dentistry, University of Sciences, Techniques and Technologies of Bamako, Bamako, Mali. ${ }^{3}$ Department of International Health, Johns Hopkins Bloomberg School of Public Health, Baltimore, MD, USA.

Received: 4 July 2020 Accepted: 11 May 2021

Published online: 22 May 2021

\section{References}

1. World Health Organization. World malaria report 2020: 20 years of global progress \& challenges. Geneva, World Health Organization; 2021. https:// www.who.int/publications-detail-redirect/9789240015791

2. Schantz-Dunn J, Nour NM. Malaria and pregnancy: a global health perspective. Rev Obstet Gynecol. 2009;2:186.

3. Desai M, ter Kuile FO, Nosten F, McGready R, Asamoa K, Brabin B, et al. Epidemiology and burden of malaria in pregnancy. Lancet Infect Dis. 2007;7:93-104.

4. WHO. Intermittent preventive treatment in pregnancy (IPTp). Geneva: World Health Organization; 2018. http://www.who.int/malaria/areas/ preventive_therapies/pregnancy/en/

5. Programme National de Lutte contre le Paludisme (PNLP). Plan Stratégique de Lutte contre le Paludisme 2013-2017. Bamako, 2013. https:// www.severemalaria.org/sites/www.severemalaria.org/files/content/attac hments/2017-07-25/Mali\%20malaria\%20PStrag\%202013-17PNLP_0.pdf

6. $\mathrm{WHO}$. Updated $\mathrm{WHO}$ policy recommendation: intermittent preventive treatment of malaria in pregnancy using sulfadoxine-pyrimethamine (IPTp-SP). Geneva: World Health Organization; 2014. http://www.who.int/ malaria/publications/atoz/iptp-sp-updated-policy-brief-24jan2014-an. pdf

7. Yaya S, Uthman OA, Amouzou A, Bishwajit G. Use of intermittent preventive treatment among pregnant women in sub-Saharan Africa: evidence from malaria indicator surveys. Trop Med Infect Dis. 2018;3:18.

8. WHO. World Malaria Report 2014. Geneva: World Health Organization; 2014. https://www.who.int/malaria/publications/world_malaria_report_ 2014/report/en/

9. Programme Nationale de Lutte contre le Paludisme. Politique Nationale de lutte contre le Paludisme 2011-2015. Bamako: PNLP; 2011.

10. CPS/SSDSPF, INSTAT, INFO-STAT. Enquête Démographique et de Santé au Mali 2012-2013. ICF International, Rockville, Maryland, USA; 2014. http:// www.sante.gov.ml/index.php/annuaires/send/8-enquetes-demographi ques-de-sante/4-eds-v-2013

11. Direction Régionale de la Santé, SLIS. Annuaire statistique 2016 de la Région de Sikasso. Sikasso; 2017.

12. Hurley EA, Harvey SA, Rao N, Diarra NH, Klein MC, Diop SI, et al. Underreporting and missed opportunities for uptake of intermittent preventative treatment of malaria in pregnancy (IPTp) in Mali. PLoS ONE. 2016;11:0160008.

13. Programme National de Lutte contre le Paludisme (PNLP), Institut National de la Statistique (INSTAT), INFO-STAT, Institut National de la Recherche en Santé Publique (INRSP), ICF International. Enquête sur les Indicateurs du Paludisme au Mali (EIPM) 2015. Rockville, Maryland, USA: INSTAT, INFO-STAT and ICF International; 2016. https://www.dhsprogram. com/pubs/pdf/MIS24/MIS24.pdf

14. Institut National de la Statistique (INSTAT), Cellule de Planification et de Statistique Secteur Santé-Développement Social et Promotion de la
Famille (CPS/SS-DS-PF), ICF. Enquête Démographique et de Santé au Mali 2018. Bamako, Mali et Rockville, Maryland, USA: INSTAT, CPS/SSDS-PF and ICF; 2019. https://www.dhsprogram.com/pubs/pdf/FR358/FR358.pdf

15. Klein MC, Harvey SA, Diarra H, Hurley EA, Rao N, Diop S, et al. "There is no free here, you have to pay": actual and perceived costs as barriers to intermittent preventive treatment of malaria in pregnancy in Mali. Malar J. 2016;15:158.

16. Hill J, Kayentao K, Toure M, Diarwara S, Bruce J, Smedley J, et al. Effectiveness of antenatal clinics to deliver intermittent preventive treatment and insecticide treated nets for the control of malaria in pregnancy in Mali: a household survey. PLoS ONE. 2014;9:e92102.

17. Webster J, Kayentao K, Bruce J, Diawara SI, Abathina A, Haiballa AA, et al. Prevention of malaria in pregnancy with intermittent preventive treatment and insecticide treated nets in Mali: a quantitative health systems effectiveness analysis. PLOS ONE. 2013;8:e67520.

18. Webster J, Kayentao K, Diarra S, Diawara SI, Haiballa AA, Doumbo OK, et al. A qualitative health systems effectiveness analysis of the prevention of malaria in pregnancy with intermittent preventive treatment and insecticide treated nets in Mali. PLOS ONE. 2013;8:e65437.

19. Sangaré LR, Stergachis $A$, Brentlinger PE, Richardson BA, Staedke SG, Kiwuwa MS, et al. Determinants of use of intermittent preventive treatment of malaria in pregnancy: Jinja Uganda. PLOS ONE. 2010;5:e15066.

20. Hill J, Dellicour S, Bruce J, Ouma P, Smedley J, Otieno P, et al. Effectiveness of antenatal clinics to deliver intermittent preventive treatment and insecticide treated nets for the control of malaria in pregnancy in Kenya. PLOS ONE. 2013;8:e64913.

21. Faye A, Manga NM, Seck I, Niang K, Leye MM, Diagne-Camara M, et al. [Access to intermittent preventive treatment (IPT) in a situation of abolition of user's fee: role of economic welfare](in French). Bull Soc Pathol Exot. 1990;2012(105):215-9.

22. Florey L. Preventing malaria during pregnancy in Sub-Saharan Africa: determinants of effective IPTp delivery. DHS analytical studies No. 39. Calverton, Maryland, USA: ICF International; 2013. https://dhsprogram. com/pubs/pdf/AS39/AS39.pdf.

23. van Eijk AM, Hill J, Larsen DA, Webster J, Steketee RW, Eisele TP, et al. Coverage of intermittent preventive treatment and insecticide-treated nets for the control of malaria during pregnancy in sub-Saharan Africa: a synthesis and meta-analysis of national survey data, 2009-11. Lancet Infect Dis. 2013;13:1029-42.

24. Hill J, Hoyt J, van Eijk AM, D'Mello-Guyett L, ter Kuile FO, Steketee R, et al. Factors affecting the delivery, access, and use of interventions to prevent malaria in pregnancy in sub-Saharan Africa: a systematic review and meta-analysis. PLoS Med. 2013;10:e1001488.

25. Ameh S, Owoaje E, Oyo-Ita A, Kabiru CW, Akpet OE, Etokidem A, et al. Barriers to and determinants of the use of intermittent preventive treatment of malaria in pregnancy in Cross River State, Nigeria: a cross-sectional study. BMC Pregnancy Childbirth. 2016;16:99.

26. Doku DT, Zankawah MM, Adu-Gyamfi AB. Factors influencing dropout rate of intermittent preventive treatment of malaria during pregnancy. BMC Res Notes. 2016;9:460.

27. Kibusi SM, Kimunai E, Hines CS. Predictors for uptake of intermittent preventive treatment of malaria in pregnancy (IPTp) in Tanzania. BMC Public Health. 2015:15:540.

28. Leonard N, Eric FB, Judith AK, Samuel W. Factors associated to the use of insecticide treated nets and intermittent preventive treatment for malaria control during pregnancy in Cameroon. Arch Public Health. 2016; 74: 5.

29. Muhumuza E, Namuhani N, Balugaba BE, Namata J, Kiracho EE. Factors associated with use of malaria control interventions by pregnant women in Buwunga subcounty. Bugiri District Malar J. 2016;15:342.

30. Rassi C, Graham K, King R, Ssekitooleko J, Mufubenga P, Gudoi SS. Assessing demand-side barriers to uptake of intermittent preventive treatment for malaria in pregnancy: a qualitative study in two regions of Uganda. Malar J. 2016;15:530.

31. Rassi C, Graham K, Mufubenga P, King R, Meier J, Gudoi SS. Assessing supply-side barriers to uptake of intermittent preventive treatment for malaria in pregnancy: a qualitative study and document and record review in two regions of Uganda. Malar J. 2016;15:341.

32. Centre de Santé de Référence. Carte Sanitaire 2012 du District Sanitaire de Sélingué. Système Local d'Information Sanitaire (SLIS); 2012.

33. Wikipedia, the free encyclopedia. Sankarani River. http://en.wikipedia. org/wiki/Sankarani_River 
34. Centre de Santé de Référence. Tableau de bord et PARAD 2014 de Sélingué. Système Local d'Information Sanitaire (SLIS); 2015.

35. Magpi. Advanced Mobile Data Collection and Visualization. https://home. magpi.com

36. SPSS Statistics 20 Available for Download. 2014. https://www.ibm.com/ support/pages/spss-statistics-20-available-download.

37. Laerd Statistics. Kendall's Tau-b using SPSS Statistics. 2013. https://stati stics.laerd.com/spss-tutorials/kendalls-tau-b-using-spss-statistics.php

38. Shaw R, Ainsworth M. Financing health services through user fees and insurance, case studies from Sub-Saharan Africa. Washington: World Bank; 1995.

39. WHO. Equity. Geneva, World Health Organization, 2014. http://www.who. int/healthsystems/topics/equity/en/

40. Kunst AE, Houweling T. A global picture of poor-rich differences in the 10. Utilization of delivery care. Studies in Health Services Organization and Policy. 2001. https://www.researchgate.net/publication/254733871_A_ global_picture_of_poor-rich_differences_in_the_utilisation_of_delivery_ care

41. Graham WJ, Fitzmaurice AE, Bell JS, Cairns JA. The familial technique for linking maternal death with poverty. Lancet. 2004;363:23-7.

42. Zere E, Tumusiime P, Walker O, Kirigia J, Mwikisa C, Mbeeli T. Inequities in utilization of maternal health interventions in Namibia: implications for progress towards MDG 5 targets. Int J Equity Health. 2010;9:16.

43. El-Khoury M, Hatt L, Gandaho T. User fee exemptions and equity in access to caesarean sections: an analysis of patient survey data in Mali. Int J Equity Health. 2012;11:49.

44. Rutstein SO, Johnson K. The DHS Wealth Index: DHS Comparative Reports [Internet]. Calverton, Maryland USA; 2004. https://dhsprogram. com/pubs/pdf/cr6/cr6.pdf

45. Hossain MI. Inequality in the utilization of maternal care and the impact of a macroeconomic policy: evidence from Bangladesh. HEDG Working Paper. 2010;10:8.

46. Wagstaff A, Van Doorslaer E. Measuring and testing for inequity in the delivery of health care. J Hum Resour. 2000;35:716-33.

47. Ndyomugyenyi R, Katamanywa J. Intermittent preventive treatment of malaria in pregnancy (IPTp): do frequent antenatal care visits ensure access and compliance to IPTp in Ugandan rural communities? Trans $\mathrm{R}$ Soc Trop Med Hyg. 2010;104:536-40.

48. Odjidja E, Kwanin C. Low uptake of intermittent preventive treatment in Ghana: an examination of health system bottlenecks. Health Syst Policy Res. 2017; 4: 58.

49. Ibrahim H, Maya ET, Issah K, Apanga PA, Bachan EG, Noora CL. Factors influencing uptake of intermittent preventive treatment of malaria in pregnancy using sulphadoxine pyrimethamine in Sunyani Municipality Ghana. Pan Afr Med J. 2017;28:122.

50. Chico RM, Dellicour S, Roman E, Mangiaterra V, Coleman J, Menendez C, et al. Global Call to Action: maximize the public health impact of intermittent preventive treatment of malaria in pregnancy in sub-Saharan Africa. Malar J. 2015;14:207.

51. Kiwuwa MS, Mufubenga P. Use of antenatal care, maternity services, intermittent presumptive treatment and insecticide treated bed nets by pregnant women in Luwero district. Uganda Malar J. 2008;7:44.
52. Doumbia S, Fondjo E, Halidou M, Diop S, Sangho O. Rapport technique final Projet « Le contrôle intégré du paludisme basé sur une base environnementale et communautaire dans les agrosystèmes de l'Afrique de I'Ouest ». Bamako; 2013.

53. Centre de Santé de Référence de Sélingué. Rapport Mensuel d'Activités (RMA) 2014 des sites ASC. Selingué: Système Local d'Information Sanitaire (SLIS); 2015.

54. Simkhada B, van Teijlingen ER, Porter M, Simkhada P. Factors affecting the utilization of antenatal care in developing countries: systematic review of the literature. J Adv Nurs. 2008;61:244-60.

55. Gabrysch S, Campbell OM. Still too far to walk: literature review of the determinants of delivery service use. BMC Pregnancy Childbirth. 2009;9:34.

56. Taylor YJ, Laditka SB, Laditka JN, Huber LRB, Racine EF. Associations of household wealth and individual literacy with prenatal care in ten West African countries. Matern Child Health J. 2016;20:2402-10.

57. Yaya S, Kota K, Buh A, Bishwajit G. Antenatal visits are positively associated with uptake of tetanus toxoid and intermittent preventive treatment in pregnancy in Ivory Coast. BMC Public Health. 2019;19:1467.

58. Mushi V, Mbotwa CH, Zacharia A, Ambrose T, Moshi FV. Predictors for the uptake of optimal doses of sulfadoxine-pyrimethamine for intermittent preventive treatment of malaria during pregnancy in Tanzania: further analysis of the data of the 2015-2016 Tanzania demographic and health survey and malaria indicator survey. Malar J. 2021;20:75.

59. Onyeneho NG, Orji BC, Okeibunor JC, Brieger W. Characteristics of Nigerian women taking sulfadoxine/pyrimethamine twice during pregnancy for the prevention of malaria. Int Gynaecol Obstet. 2013;123:101-4.

60. Pell C, Straus L, Andrew EV, Meñaca A, Pool R. Social and cultural factors affecting uptake of interventions for malaria in pregnancy in Africa: a systematic review of the qualitative research. PLOS ONE. 2011;6:e22452.

61. Mubyazi G, Bloch P, Kamugisha M, Kitua A, ljumba J. Intermittent preventive treatment of malaria during pregnancy: a qualitative study of knowledge, attitudes and practices of district health managers, antenatal care staff and pregnant women in Korogwe District North-Eastern Tanzania. Malar J. 2005:4:31

62. Mubyazi GM, Magnussen P, Goodman C, Bygbjerg IC, Kitua AY, Olsen $\varnothing \mathrm{E}$, et al. Implementing intermittent preventive treatment for malaria in pregnancy: review of prospects, achievements, challenges and agenda for research. Open Trop Med J. 2008;1:92.

63. Mbonye AK, Yanow S, Birungi J, Magnussen P. A new strategy and its effect on adherence to intermittent preventive treatment of malaria in pregnancy in Uganda. BMC Pregnancy Childbirth. 2013;13:178.

64. PNLP, MSHP/Mali. Directives Nationales sur la gestion et la distribution gratuite des Moustiqueaires Imprégnées d'insectivide de longue durée ; de la sulfadoxine pyriméthamine et la chimioprévention du paludisme saisonnier. Bamako, 2016

\section{Publisher's Note}

Springer Nature remains neutral with regard to jurisdictional claims in published maps and institutional affiliations.

Ready to submit your research? Choose BMC and benefit from

- fast, convenient online submission

- thorough peer review by experienced researchers in your field

- rapid publication on acceptance

- support for research data, including large and complex data types

- gold Open Access which fosters wider collaboration and increased citations

- maximum visibility for your research: over $100 \mathrm{M}$ website views per year

At BMC, research is always in progress.

Learn more biomedcentral.com/submissions 